\section{Disappearance of slan-positive non-classical monocytes for diagnosis of chronic myelomonocytic leukemia with an associated inflammatory state}

The diagnosis of chronic myelomonocytic leukemia (CMML) relies on both a persistent peripheral blood monocytosis (monocytes $\geq 1 \times 10^{9} / \mathrm{L}$ ) and monocytes accounting for $\geq 10 \%$ of the white blood cell (WBC) differential count. ${ }^{1}$ We showed that a relative accumulation of classical monocytes (cMo: CD $14^{++} \mathrm{CD} 16^{-}$) $\geq 94 \%$ among the total peripheral blood monocytes measured by flow cytometry could help to distinguish CMML from other causes of monocytosis. ${ }^{2}$ Since then, the quantification of circulating monocyte subsets for the diagnosis of this disease has become widespread. ${ }^{3-7}$ The increase in cMo is related to a decrease in the fraction of non-classical monocytes (ncMo: CD14 $\left.{ }^{- \text {low }} \mathrm{CD} 16^{+}\right)^{2,5}$

This flow cytometry criterion can be challenged by the co-occurrence of an inflammatory disease, observed in up to $20 \%$ of CMML patients depending on the series studied, or an inflammatory state. ${ }^{8}$ Inflammation can provoke an increase in the intermediate subset of monocytes (iMo: $\mathrm{CD} 14^{++} \mathrm{CD} 16^{+}$) with a concomitant decrease in the relative cMo percentage below the typical $94 \%$ threshold, erasing the CMML signature and generating an easily recognizable "bulbous" profile. ${ }^{6}$ As the CMML-associated decrease in the ncMo fraction persists in an inflammatory setting, ${ }^{3,6}$ it might be a relevant criterion for differentiating CMML from a reactive monocytosis, pending an accurate delineation between iMo and ncMo subsets. ${ }^{9}$ At present, routine use of a decreased ncMo fraction in diagnostics is precluded by the lack of inter-operator reproducibility of its measurement, unlike cMo fraction measurement.

Slan, also known as 6-sulfo LacNac, a carbohydrate modification of P-selectin glycoprotein ligand 1, is considered to be a marker of ncMo. ${ }^{10}$ To improve the flow cytometry distinction between CMML and reactive monocytosis, we analyzed the utility of measuring the slan-positive $\left(\mathrm{slan}^{+}\right)$ncMo fraction in the peripheral blood of CMML patients, especially those with a concomitant inflammatory state.

We first analyzed, by flow cytometry, slan expression at the surface of circulating blood cells in samples collected from 22 young (mean age: $38 \pm 13$ years) and 35 elderly (mean age: $76 \pm 8$ years) healthy donors. The mean absolute monocyte count (AMC) of the young individuals was $0.51 \pm 0.19 \times 10^{9} / \mathrm{L}$. while that of the older subjects was $\left.0.6 \pm 0.15 \times 10^{9} / \mathrm{L}\right)$. Whole blood samples were stained as described elsewhere ${ }^{3}$ with anti-CD45, CD2, CD56, CD24, CD14, CD16 (Beckman-Coulter or BDBiosciences) and anti-slan (Miltenyi-Biotec) antibodies and analyzed with either a Navios (Beckman Coulter) or Fortessa (BDBiosciences) cytometer. Regardless of age, slan was detected only at the surface of monocytes $(6.5 \pm 3.2 \%)$, not being expressed on neutrophils $(0.05 \pm 0.2 \%)$ or lymphocytes $(0.3 \pm 0.2 \%)$, including natural killer cells $(0.1 \pm 0.2 \%)$ (Figure $1 \mathrm{~A})$. Following an exclusion gating strategy, monocytes were separated into cMo $\left(\mathrm{CD} 14^{++} \mathrm{CD} 16\right)$, iMo $\left(\mathrm{CD} 14^{++} \mathrm{CD} 16^{+}\right)$and ncMo $\left(\mathrm{CD} 14 /^{\text {low }} \mathrm{CD} 16^{+}\right)$subsets. $^{2}$ The expression of slan was observed to be mostly restricted to ncMo, with $95.7 \pm 3.4 \%$ of $\operatorname{slan}^{+}$cells gathering within this subpopulation (Figure 1B, C).

To ensure a reproducible definition of slan ${ }^{+}$cells among monocyte subsets, we chose a slan/CD16 dot plot representation, which allowed an accurate definition of the threshold for slan positivity, determined against the cMo population (Figure 1C). Only a fraction of ncMo $(51.7 \pm 12.0 \%)$ were $\operatorname{slan}^{+}$, corresponding to monocytes with the weakest expression of CD14. Slan ${ }^{+}$and slannegative ncMo subpopulations displayed similar morphological features (Figure 1D). These results confirmed that slan is specifically expressed by a fraction of ncMo.

Between November 2017 and October 2018, we collected peripheral blood samples or peripheral blood mononucleated cells from a learning cohort of subjects consisting of 26 patients with a reactive monocytosis, 55 patients with newly diagnosed CMML (diagnosis made according to World Health Organization criteria $\left.{ }^{1}\right)$ and 72 healthy donors (37 young and 35 elderly) in two centers (Gustave Roussy and Henri Mondor University Hospitals) following approval by the Ile-de-France 1 (DC2014-2091) and the Groupe Francophone des Myélodysplasies ethical committees (Table 1). The CMML patients had a mean age of $75 \pm 9$ years, with a typical male predominance (male to female ratio, 2.5:1) and $\mathrm{WBC}$ count ranging between $3 \times 10^{9} / \mathrm{L}$ and $116 \times 10^{9} / \mathrm{L}$, Eighteen of these patients $(33 \%)$ were classified as having proliferative CMML $\left(\mathrm{WBC} \geq 13 \times 10^{9} / \mathrm{L}\right) .{ }^{11}$ Their AMC ranged from $1.0 \times 10^{9} / \mathrm{L}$ to $40.7 \times 10^{9} / \mathrm{L}$ with a mean monocyte percentage of $28.4 \pm 12.7 \%$. Bone marrow analysis confirmed dysplasia in $96 \%$ of the samples (multilineage dysplasia in $81 \%$ ) and allowed subdivision of these patients into 18 with CMML-0 (33\%), 28 with CMML-1 (51\%), and 9 with CMML-2 (16\%). These patients had slight decreases of both hemoglobin concentration and platelet count. CMML-2 patients showed a significantly deeper thrombocytopenia compared to CMML-1 and CMML-0 patients $(P<0.01)$. Patients with reactive monocytosis were younger $(65 \pm 14$ years) than CMML patients; they had similar mean WBC counts but lower AMC (range: $1 \times 10^{9} / \mathrm{L}-8.9 \times 10^{9} / \mathrm{L}$ ). The male to female ratio was 1.9:1 and these patients presented with various causal diseases including systemic autoimmune and/or inflammatory disease, acute infection, metastatic neoplasm, and diffuse lymphoma.

We analyzed monocyte subset distribution in this learning cohort. Forty-eight of the $55 \mathrm{CMML}$ patients had $\mathrm{cMo} \geq 94 \%$ (Figure $1 \mathrm{E}$ ), in accordance with previous series. $^{2-7}$ The flow cytometry profile of all the seven CMML patients whose cMo percentage was below $94 \%$ had an easily recognized "bulbous" appearance (Figure 1F) that has been associated with an inflammatory state ${ }^{6}$ (C-reactive protein level, available for 4 of these 7 patients: $68.3 \pm 80.5 \mathrm{mg} / \mathrm{L})$. This particular profile was due to an increase in the iMo fraction $(12.4 \pm 7.3 \%)$, in comparison to the fraction present in the CMML patients without an inflammatory state $(2.0 \pm 1.0 \%)$, combined with the disappearance of the ncMo subset (Table 1).

A decrease of the ncMo fraction below $1.13 \%$ of total circulating monocytes was recently proposed to identify CMML (Figure 1G). ${ }^{6}$ This characteristic feature of the disease was shown to involve the epigenetic deregulation of a miR-150/TET3 pathway that prevents the conversion of cMo into ncMo. ${ }^{12}$ The quantification of ncMo requires a robust delineation between iMo and ncMo. This latter subpopulation was first defined by CD14 expression level $;^{13}$ subsequently, this strategy was refined using an oblique line to gate the population. ${ }^{9}$ Discrepancies between ncMo percentages determined by these two strategies are illustrated in Figure $1 \mathrm{H}$, I, which highlights the difficulty in accurately and reproducibly quantifying the ncMo subset. Using the oblique line strategy, the relative fraction of ncMo was significantly lower in CMML patients $(1.6 \pm 1.3 \%)$ than in controls $(11.4 \pm 4.9 \%)$ or in patients with reactive monocytosis $(10.1 \pm 5.4 \%)$ (Figure 
Table 1. Clinical and biological parameters in patients with chronic myelomonocytic leukemia or a reactive monocytosis.

\begin{tabular}{|c|c|c|c|c|c|c|c|}
\hline & All CMML & CMML-0 & CMML-1 & $\begin{array}{l}\text { RNING COP } \\
\text { CMML-2 }\end{array}$ & $\begin{array}{l}\text { CMML } \\
\text { with } \\
\text { bullbous } \\
\text { aspect }\end{array}$ & $\begin{array}{l}\text { CMML } \\
\text { without } \\
\text { bullbous } \\
\text { aspect }\end{array}$ & $\begin{array}{l}\text { Reactive } \\
\text { monocytosis }\end{array}$ \\
\hline Patients, n (\%) & $55(100 \%)$ & $18(33 \%)$ & $28(51 \%)$ & $9(16 \%)$ & $7(13 \%)$ & $48(87 \%)$ & $26(100 \%)$ \\
\hline Ratio male/ female & 2.5 & 5.0 & 3.3 & 0.5 & 2.0 & 2.6 & 1.9 \\
\hline Age (years) & $75 \pm 9$ & $76 \pm 11$ & $75 \pm 9$ & $75 \pm 8$ & $73 \pm 7$ & $75 \pm 10$ & $65 \pm 14$ \\
\hline CBC, n (\%) & $55(100 \%)$ & & & & & & $26(100 \%)$ \\
\hline $\mathrm{Hb}, \mathrm{g} / \mathrm{dL}$ & $11.8 \pm 2.4$ & $11.97 \pm 3.1$ & $12.2 \pm 2.0$ & $10.3 \pm 1.8$ & $9.1 \pm 2.3$ & $12.1 \pm 2.3$ & $11.8 \pm 2.6$ \\
\hline Platelets, x10/L & $159 \pm 123$ & $199 \pm 134$ & $166 \pm 117$ & $58 \pm 37$ & $185 \pm 144$ & $152 \pm 120$ & $275 \pm 151$ \\
\hline $\mathrm{WBC}, \times 10^{9} / \mathrm{L}$ & $16.6 \pm 23.8$ & $14.3 \pm 25.4$ & $17.3 \pm 25.0$ & $19.0 \pm 18.4$ & $28.1 \pm 40.0$ & $14.8 \pm 19.5$ & $14.6 \pm 8.3$ \\
\hline ANC, $x 10^{9} / \mathrm{L}$ & $8.0 \pm 15.7$ & $10.6 \pm 24.7$ & $6.2 \pm 7.9$ & $34.8 \pm 6.7$ & $8.6 \pm 12.0$ & $8.1 \pm 16.1$ & $10.6 \pm 8.0$ \\
\hline $\mathrm{AMC}, \mathrm{x} 10^{9} / \mathrm{L}$ & $4.1 \pm 6.7$ & $2.3 \pm 1.5$ & $4.6 \pm 8.2$ & $6.2 \pm 7.8$ & $8.4 \pm 14.4$ & $3.6 \pm 4.6$ & $1.8 \pm 1.6$ \\
\hline Blood monocytes (\%) & $28.4 \pm 12.7$ & $24.8 \pm 10.7$ & $28.6 \pm 8.5$ & $36.0 \pm 22.6$ & $35.6 \pm 6.3$ & $27.5 \pm 13.1$ & $12.2 \pm 5.4$ \\
\hline Marrow analysis, $\mathrm{n}(\%)$ & $55(100 \%)$ & & $55(100 \%)$ & & $55(100 \%)$ & & NA \\
\hline Marrow monocytes (\%) & $11.3 \pm 6.5$ & $9.2 \pm 6.1$ & $13.3 \pm 6.8$ & $9.1 \pm 3.6$ & $11.7 \pm 6.1$ & $11.2 \pm 6.6$ & NA \\
\hline Marrow blasts (\%) & $6.8 \pm 3.9$ & $2.8 \pm 0.9$ & $6.9 \pm 1.3$ & $13.7 \pm 2.3$ & $5.9 \pm 4.4$ & $6.9 \pm 3.8$ & $\mathrm{NA}$ \\
\hline Dysplasia, n & $52 / 54$ & $18 / 18$ & $25 / 27$ & $9 / 9$ & $7 / 7$ & $45 / 47$ & NA \\
\hline Multilineage dysplasia, $n$ & $44 / 54$ & $14 / 18$ & $23 / 27$ & $7 / 9$ & $7 / 7$ & $37 / 47$ & \\
\hline Monocyte assay, n (\%) & $55(100 \%)$ & & & & & & $26(100 \%)$ \\
\hline cMo (\%) & $95.2 \pm 5.0$ & $93.8 \pm 5.9$ & $95.6 \pm 5.0$ & $96.5 \pm 2.8$ & $84.5 \pm 7.9$ & $96.7 \pm 1.4$ & $82.9 \pm 8.4$ \\
\hline iMo (\%) & $3.3 \pm 4.4$ & $3.9 \pm 4.9$ & $3.0 \pm 4.5$ & $2.8 \pm 2.3$ & $12.4 \pm 7.3$ & $1.9 \pm 1.0$ & $7.1 \pm 5.4$ \\
\hline ncMo (\%) & $1.6 \pm 1.3$ & $2.3 \pm 1.7$ & $1.4 \pm 1.0$ & $0.7 \pm 0.6$ & $3.2 \pm 1.9$ & $1.3 \pm 1.1$ & $10.1 \pm 5.4$ \\
\hline ncMo slan ${ }^{+}(\%)$ & $0.4 \pm 0.5$ & $0.6 \pm 0.1$ & $0.3 \pm 0.4$ & $0.1 \pm 0.1$ & $0.5 \pm 0.5$ & $0.4 \pm 0.5$ & $4.3 \pm 3.0$ \\
\hline
\end{tabular}

\begin{tabular}{|c|c|c|c|c|c|c|c|}
\hline & All CMML & CMML-O & CMML-1 & $\begin{array}{l}\text { DATION COH } \\
\text { CMML-2 }\end{array}$ & $\begin{array}{l}\text { CMML } \\
\text { with } \\
\text { bulbous } \\
\text { aspect }\end{array}$ & $\begin{array}{l}\text { CMML } \\
\text { without } \\
\text { bulbous } \\
\text { aspect }\end{array}$ & $\begin{array}{c}\text { Reactive } \\
\text { monocytosis }\end{array}$ \\
\hline Patients, n (\%) & $36(100 \%)$ & $20(56 \%)$ & $10(28 \%)$ & $6(17 \%)$ & $5(14 \%)$ & $31(86 \%)$ & $22(100 \%)$ \\
\hline Ratio male/ female & 3.5 & 5.7 & 2.3 & 2.0 & 4.0 & 3.4 & 2.7 \\
\hline Age (years) & $71 \pm 12$ & $73 \pm 11$ & $68 \pm 11$ & $67 \pm 18$ & $70 \pm 3$ & $71 \pm 13$ & $47 \pm 18$ \\
\hline CBC, n (\%) & $36(100 \%)$ & & & & & & $22(100 \%)$ \\
\hline $\mathrm{Hb}, \mathrm{g} / \mathrm{dL}$ & $11.5 \pm 1.7$ & $11.5 \pm 1.6$ & $12.0 \pm 1.5$ & $10.8 \pm 2.0$ & $10.8 \pm 2.3$ & $11.6 \pm 1.6$ & $11.2 \pm 2.4$ \\
\hline Platelets, $\times 10^{9} / \mathrm{L}$ & $158 \pm 183$ & $201 \pm 233$ & $113 \pm 36$ & $92 \pm 86$ & $177 \pm 165$ & $155 \pm 188$ & $309 \pm 175$ \\
\hline $\mathrm{WBC}, \times 10^{9} / \mathrm{L}$ & $20.0 \pm 22.4$ & $22.7 \pm 25.9$ & $10.5 \pm 10.2$ & $26.8 \pm 22.7$ & $29.8 \pm 15.9$ & $18.4 \pm 23.1$ & $14.9 \pm 3.9$ \\
\hline ANC, $\times 10^{9} / \mathrm{L}$ & $11.5 \pm 14.7$ & $15.3 \pm 18.0$ & $6.3 \pm 7.7$ & $6.8 \pm 2.2$ & $19.6 \pm 11.6$ & $10.1 \pm 14.9$ & $10.4 \pm 4.4$ \\
\hline $\mathrm{AMC}, \times 10^{9} / \mathrm{L}$ & $3.4 \pm 4.3$ & $3.0 \pm 3.9$ & $2.2 \pm 1.5$ & $7.6 \pm 7.4$ & $3.0 \pm 1.5$ & $3.5 \pm 4.6$ & $1.5 \pm 0.4$ \\
\hline Blood monocytes (\%) & $21.4 \pm 9.6$ & $18.5 \pm 9.6$ & $24.4 \pm 8.9$ & $28.9 \pm 6.9$ & $13.1 \pm 9.2$ & $22.9 \pm 9.1$ & $10.3 \pm 2.9$ \\
\hline Marrow analysis, n (\%) & $36(100 \%)$ & & & & & & NA \\
\hline Marrow monocytes (\%) & $9.5 \pm 4.6$ & $8.1 \pm 3.9$ & $8.9 \pm 3.3$ & $16.2 \pm 3.6$ & $11.4 \pm 5.9$ & $9.2 \pm 4.3$ & NA \\
\hline Marrow blasts (\%) & $5.4 \pm 4.2$ & $2.6 \pm 1.2$ & $6.8 \pm 1.3$ & $12.8 \pm 3.1$ & $4.8 \pm 5.7$ & $5.5 \pm 4.0$ & \\
\hline Dysplasia, $\mathrm{n}$ & $32 / 33$ & $18 / 18$ & $8 / 9$ & $6 / 6$ & $5 / 5$ & $27 / 28$ & NA \\
\hline Multilineage dysplasia, $n$ & $20 / 33$ & $9 / 18$ & $7 / 9$ & $4 / 6$ & $2 / 5$ & $18 / 28$ & \\
\hline Monocyte assay, n (\%) & $36(100 \%)$ & & & & & & $22(100 \%)$ \\
\hline cMo (\%) & $96.3 \pm 2.7$ & $96.2 \pm 2.8$ & $96.8 \pm 1.3$ & $95.9 \pm 4.0$ & $90.7 \pm 1.8$ & $97.2 \pm 1.4$ & $84.6 \pm 9.2$ \\
\hline iMo (\%) & $2.7 \pm 2.3$ & $2.8 \pm 1.9$ & $1.9 \pm 1.2$ & $3.4 \pm 4.1$ & $7.5 \pm 2.3$ & $2.0 \pm 1.1$ & $6.8 \pm 2.9$ \\
\hline ncMo (\%) & $1.0 \pm 0.8$ & $1.0 \pm 0.8$ & $1.3 \pm 1.0$ & $0.6 \pm 0.4$ & $1.9 \pm 1.1$ & $0.9 \pm 0.7$ & $8.6 \pm 7.3$ \\
\hline ncMo slan $(\%)$ & $0.3 \pm 0.4$ & $0.3 \pm 0.4$ & $0.4 \pm 0.5$ & $0.2 \pm 0.2$ & $0.3 \pm 0.2$ & $0.3 \pm 0.4$ & $4.7 \pm 4.6$ \\
\hline
\end{tabular}

Patients with chronic myelomonocytic leukemia (CMML) were diagnosed and classified according to the 2017 World Health Organization criteria into three groups, CMML0 , CMML-1, and CMML-2. ${ }^{1}$ The presence of peripheral blood monocytosis and monocytes accounting for $\geq 10 \%$ of total leukocytes was verified. The presence of dysplasia (notably multilineage dysplasia) observed in bone marrow smears is specified. When dysplasia was not observed or data were missing, cytogenetic abnormalities or mutations were ascertained ( 3 cases in the learning cohort and 4 cases in the validation cohort). CMML patients were studied before any treatment. The parameters of CMML patients with a "bulbous" flow cytometry profile, suggesting an inflammatory state, and those without a "bulbous" profile are presented separately. The percentages of classical, intermediate, non-classical and slan ${ }^{+}$non-classical monocytes were determined among total circulating monocytes in each aforementioned subgroup of patients. All parameters are presented as the mean \pm standard deviation, unless otherwise specified.CBC: complete blood count; Hb: hemoglobin;WBC: white blood cells; ANC:absolute neutrophil count; AMC: absolute monocyte count; NA: not applicable. cMo: classical monocytes; iMo: intermediate monocytes; ncMo: non-classical monocytes. 
A

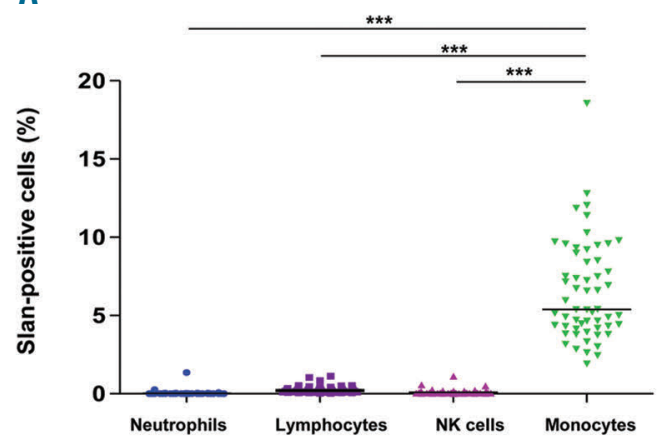

C
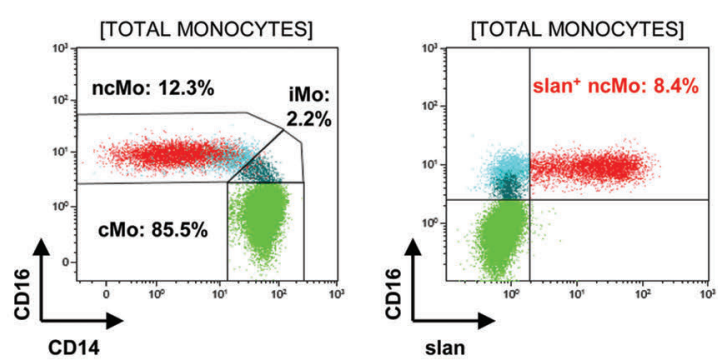

B

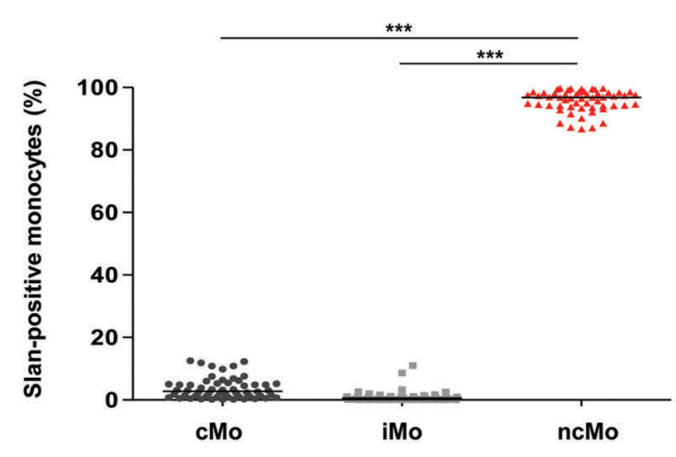

D
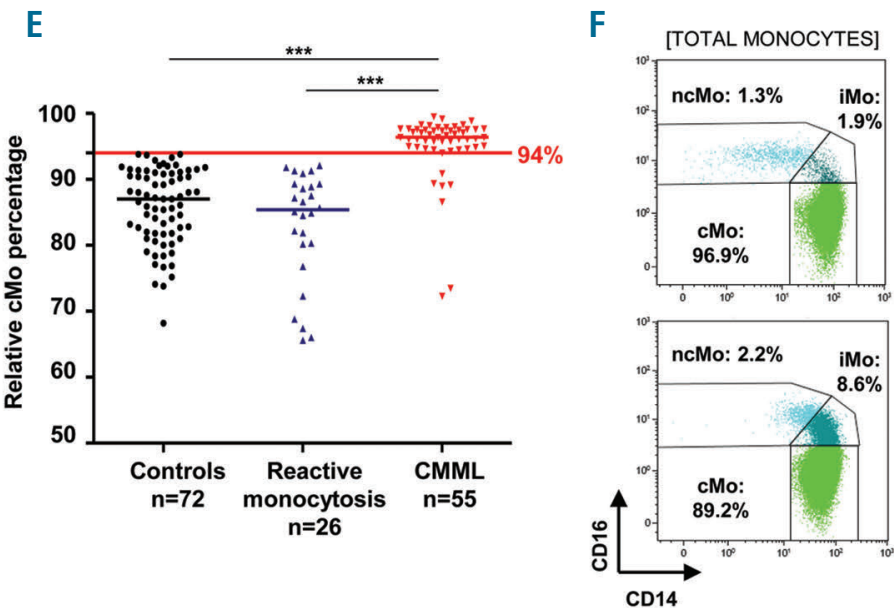

G

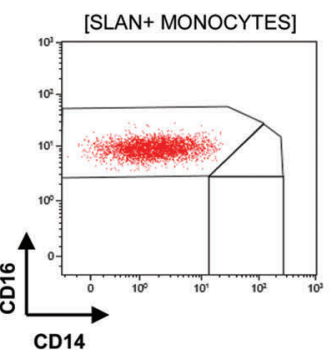

slan-negative nclMo

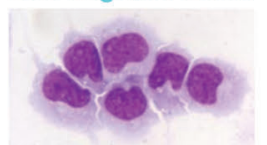

slan-positive ncMo
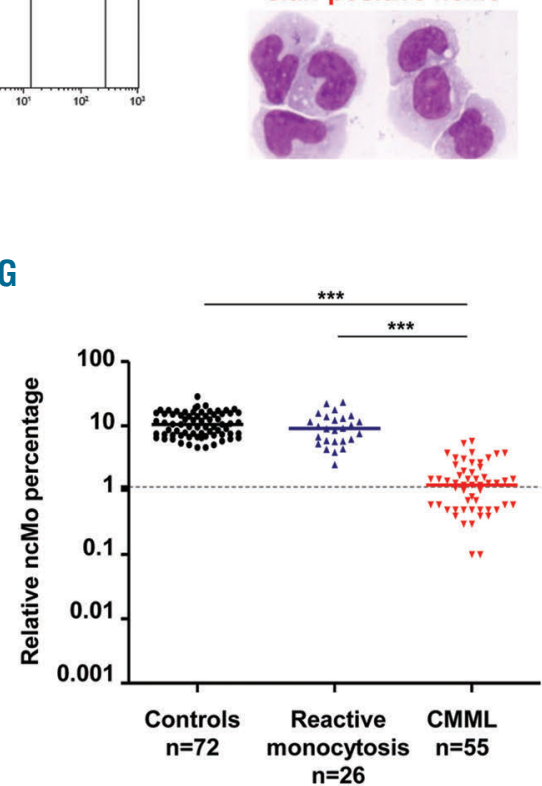

H Example of reactive monocytosis

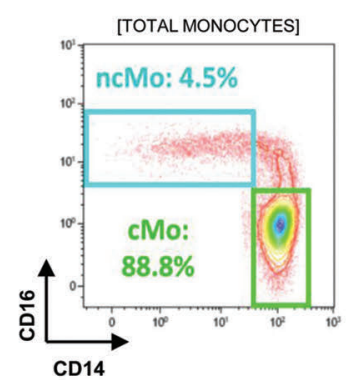

I

Example of CMML

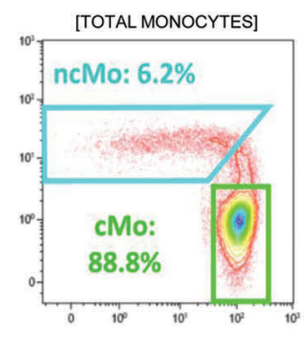

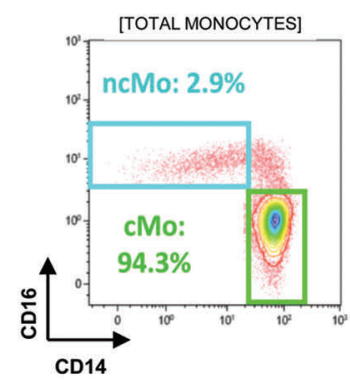




\author{
Legend to Figure 1 on previous page
}

Figure 1. Relevance of slan expression for selecting a fraction of non-classical monocytes. (A) Percentages of slan ${ }^{+}$cells in peripheral blood leukocytes from 22 healthy donors (age <65 years, Etablissement Français du Sang, Créteil and Rungis, France) and 35 aged-matched donors (age $>65$ years, Henri Mondor University Hospital). The medians are indicated by the solid black lines; $* * * P<0.001$, Mann-Whitney test. Neutrophils were defined as CD16 cells with wideranging (intermediate to high) side scatter (SSC), B lymphocytes as CD24 $4^{+} / \mathrm{SSC}^{\text {low }}$ cells and T lymphocytes as $\mathrm{CD} 7^{+}$and/or $\mathrm{CD} 2^{+} / \mathrm{CD} 56^{\text {neg }} / \mathrm{SSC} \mathrm{C}^{\text {low }}$ cells. Total monocytes were selected as previously described. ${ }^{2}$ Briefly, total monocytes were considered as the circulating cells remaining after the exclusion of both immature

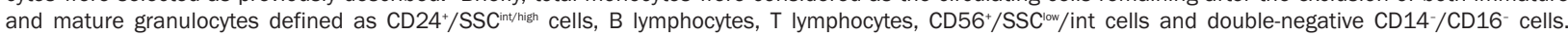
Subsequently, natural killer (NK) cells were defined as the CD45 $/$ CD56 ${ }^{+} / \mathrm{SSC}^{\text {low }}$ population, after exclusion of the above-mentioned total monocytes, in order to avoid overlapping with monocytes. Finally, total lymphocytes were defined as the sum of B lymphocytes, T lymphocytes and NK cells. (B) Percentage of slan cells in the three monocyte subsets of 22 healthy donors and 35 aged-matched donors; the medians are indicated by the solid black lines; $* * * P<0.001$, MannWhitney test. (C) Gating strategy used in a healthy donor to select monocyte subsets. Left panel: the percentages of classical (cMo), intermediate (iMo) and nonclassical (ncMo) monocytes on a CD14/CD16 dot plot are indicated. Middle panel: the percentage of slan ${ }^{+}$ncMO (shown in red) among monocytes on a slan/CD16 dot plot is shown. Right panel: representation of the selected slan+ ncM0 cells on a CD14/CD16 dot plot. (D) May-Grünwald Giemsa staining of sorted slan $^{+}$or slan- ncMO cells following a previously described gating strategy ${ }^{2}$ using Influx (BD Biosciences and BD Diva software) cell sorters (magnification x630). (E) Relative percentages of cMo among total circulating monocytes in the learning cohort consisting of 72 controls (healthy donors incremented from 22 to 37 , and 35 aged-matched donors), 26 patients with reactive monocytosis and 55 with newly-diagnosed CMML; the medians are indicated by solid lines and the $94 \%$ diagnostic threshold is indicated; $* * * P<0.001$, Mann-Whitney test. (F) Representative dot plots of monocyte subsets from a CMML patient with a typical profile (cMo >94\%) (upper panel) and a CMML patient with an inflammatory state, showing a "bulbous" profile with cMo <94\% (lower panel). (G) Relative percentages of ncMo among total circulating monocytes (determined using the refined gating strategy ${ }^{9}$ ) in the learning cohort; the medians are indicated by solid lines; the dotted line represents the ncMO threshold value proposed by Hudson and colleagues; ${ }^{*} * * P<0.001$, Mann-Whitney test. (H, I). Representative dot plots of monocyte subset repartition in a patient with a reactive monocytosis (H) and a patient with CMML (I). Since iMo and cMo express CD14 similarly, the delineation between iMo and ncMo was initially defined by CD14 expression of cMo (conventional gating). ${ }^{13}$ However, a refined gating strategy was proposed ${ }^{3}$ using an oblique line such that the entire cloud of $\mathrm{CD} 14^{++} / \mathrm{CD} 16^{+}$events is enclosed. For each example, ncMo are quantified using the conventional gating strategy (left panel) and the refined gating strategy (right panel).

1G). Nevertheless, in 31 of the $55 \mathrm{CMML}$ patients, the ncMo fraction was above the $1.13 \%$ proposed threshold. ${ }^{6}$ In other words, $24 \mathrm{CMML}$ patients with $\mathrm{cMo} \geq 94 \%$ were misclassified using the ncMo approach.

In a search for a monocyte marker whose measurement would reproducibly help to identify CMML, whatever the patient's inflammatory status, we explored the use of slan. Although this marker is mostly expressed by CD14 $4^{\text {low }}$ ncMo cells, quantification of the $\operatorname{slan}^{+}$ncMo population could be of interest in CMML. We observed that the $\operatorname{slan}^{+}$ncMo fraction was significantly lower in CMML patients $(0.4 \pm 0.5 \%)$ than in controls $(5.7 \pm 3.3 \%)$ or patients with reactive monocytosis $(4.3 \pm 3.0 \%$; $P<0.001)$ (Figure 2A-C). It is noteworthy that CMML patients who had the highest percentage of $\operatorname{slan}^{+}$ncMo were those with a clear-cut relative cMo accumulation above $94 \%$. Interestingly, CMML patients who could not be diagnosed as having CMML using the relative accumulation of cMo, because of the "bulbous" appearance of the flow cytometry profile, had a lower percentage of $\operatorname{slan}^{+}$ncMo $(0.5 \pm 0.5 \%$ for the 7 patients with CMML and an inflammatory state) (Figure 2B).

A receiver operating characteristic curve defined a cutoff value of $1.7 \%$ to identify a decrease in the peripheral blood $\operatorname{slan}^{+}$ncMo fraction (Figure 2D). Using this threshold, measurement of the $\operatorname{slan}^{+}$ncMo fraction improved the sensitivity of the diagnosis of CMML as compared to that achieved from measuring the cMo fraction (100\% vs. $86 \%$ ), since all the false negatives were retrieved, including those with an inflammatory state.

Between November 2018 and April 2019, an independent validation cohort, consisting of 22 patients with reactive monocytosis and $36 \mathrm{CMML}$ patients, including five inflammatory cases (C-reactive protein level for 3 of them: $33.6 \pm 14.1 \mathrm{mg} / \mathrm{L}$ ), was studied and similar results were obtained with a $100 \%$ sensitivity (Table 1 ). Of note, regarding the whole cohort, eight of 48 patients with a reactive monocytosis had a slan ${ }^{+}$ncMo fraction below $1.7 \%$, indicating a $83 \%$ specificity. In these cases, molecular exploration, by next-generation sequencing, of the genes most frequently mutated in CMML may be of interest. ${ }^{14}$

Serial measurements of the slan ${ }^{+}$ncMo fraction for up to 8 months in untreated CMML patients demonstrated the reproducibility of $\operatorname{slan}^{+}$ncMo $\%<1.7 \%$ over time (Figure 2E, F). We also got the opportunity to analyze this parameter in CMML patients before and after treatment with either hydroxyurea or a hypomethylating agent., ${ }^{8,15}$ Unlike hydroxyurea, hypomethylating agents were shown to restore a normal monocyte subset repartition in responding patients, ${ }^{2}$ associated with an increase of the $\operatorname{slan}^{+}$ncMo fraction over the $1.7 \%$ threshold (Figure $2 \mathrm{G})$.

The present study demonstrates that a flow cytometry analysis of monocyte subsets in the peripheral blood including the slan marker may be useful for diagnosing CMML earlier and delineating cohorts of accurately defined patients in order to evaluate the impact of treatments. Indeed, monocytosis is a common biological finding (present in about $5 \%$ of complete blood counts; data from Henri Mondor University Hospital, Créteil, France). We show here that a decrease in the specific $\operatorname{slan}^{+}$ncMo subset below $1.7 \%$ of total monocytes is a characteristic feature of CMML patients, including those with an associated inflammatory state. This decrease is stable over time in untreated patients and its disappearance may be a biomarker of response to hypomethylating drugs. We therefore propose a single flow cytometry assay and a two-step algorithm to support a diagnosis of CMML (Figure 2H). First, a cMo fraction above the 94\% threshold argues for CMML. When the cMo fraction is below $94 \%$, especially when the flow cytometry profile has a "bulbous" aspect, a $\operatorname{slan}^{+}$ncMo fraction below $1.7 \%$ argues for a CMML. Compared to genomic approaches, such a simple assay is an economically relevant strategy. ${ }^{14}$

Sihem Tarfi, ${ }^{1,2}$ Bouchra Badaoui, ${ }^{1}$ Nicolas Freynet, ${ }^{1}$ Margot Morabito, Jeffie Lafosse, ${ }^{3}$ Andréa Toma, ${ }^{4}$ Gabriel Etienne, Jean-Baptiste Micol, ${ }^{6}$ Ivan Sloma, ${ }^{1,2}$ Pierre Fenaux, ${ }^{7}$ Eric Solary, ${ }^{3,6,8}$ Dorothée Selimoglu-Buet t, $^{3,}$ and Orianne Wagner-Ballon ${ }^{1,21}$ on behalf of the Groupe Francophone des Myélodysplasies (GFM)

\section{\#joint last authorship}

'Département d'Hématologie et Immunologie Biologiques, Hôpitaux Universitaires Henri-Mondor (AP-HP), Créteil; '2INSERM U955 IMRB, UPEC, Créteil, France; INISERM U1170, Université ParisSud, Gustave Roussy Cancer Center, Villejuif; ${ }^{4}$ Service d'Hématologie 
A

Example of typical CMML
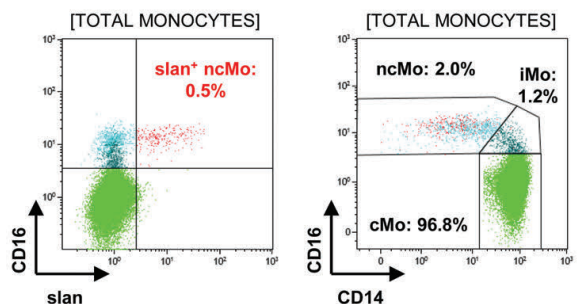

\section{Example of inflammatory CMML}
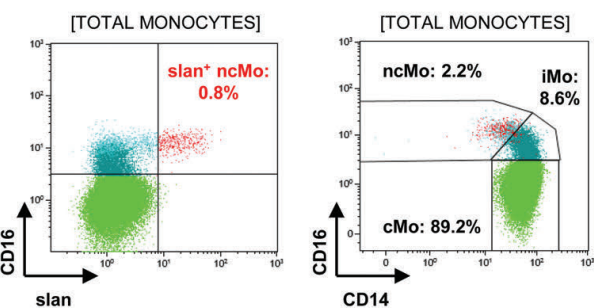

C

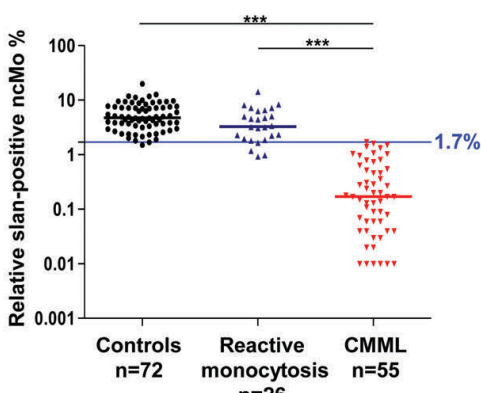

$\mathbf{F}$
D

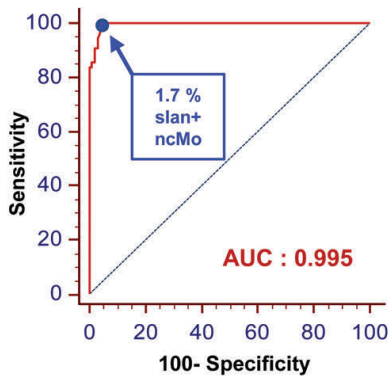

E

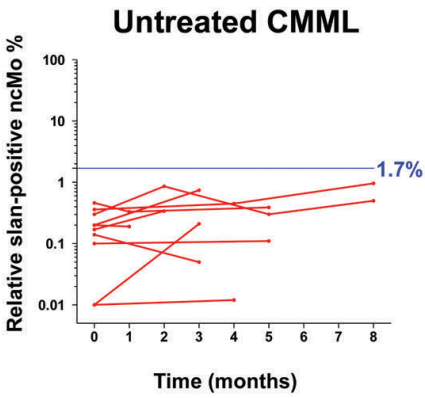

G

HMA treatment

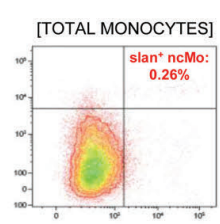

[TOTAL MONOCYTES]

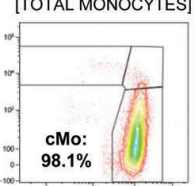

Diagnosis
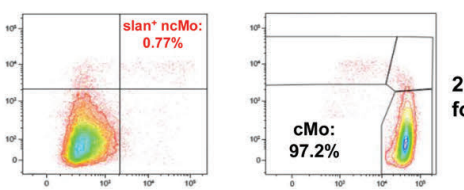

2 month

follow-up

$97.2 \%$
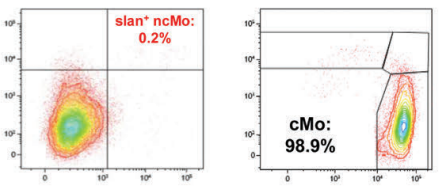

4 month

follow-up
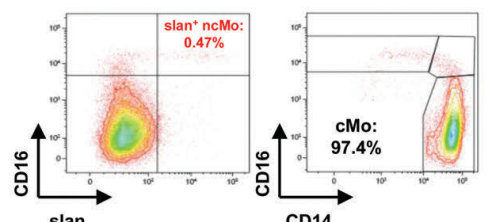

8 month

follow-up
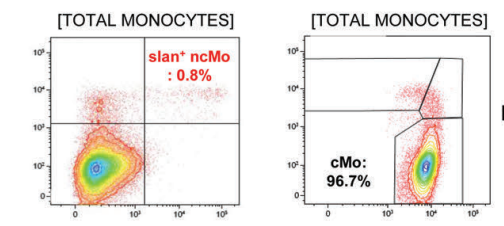

Diagnosis
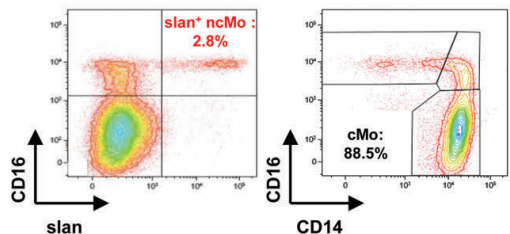

+Decitabine

(6 cycles)

H

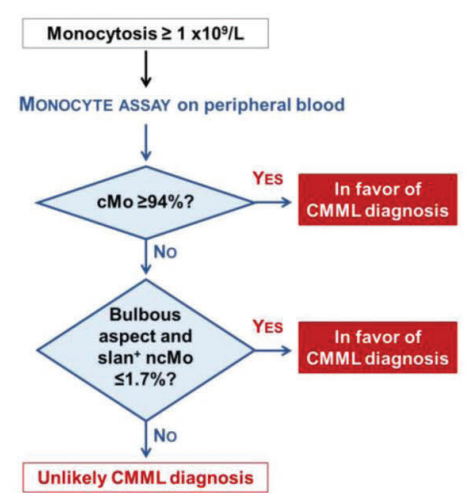

Figure 2. A characteristic decrease in the slan-positive non-classical monocyte fraction in chronic myelomonocytic leukemia. (A, B) Representative dot plots of monocytes (left panel, slan/CD16; right panel, CD14/CD16) from a patient with chronic myelomonocytic leukemia (CMML) with a typical profile (classical monocytes, cMo >94\%) (A) and a CMML patient with an inflammatory state (B), showing a "bulbous" profile with cMo <94\%. (C) Relative percentages of slan non-classical monocytes (ncMO) among total circulating monocytes in the learning cohort, consisting of 72 controls, 26 patients with reactive monocytosis and 55 patients with newly-diagnosed CMML; the medians are indicated by solid lines; $* * * P<0.001$, Mann-Whitney test. (D) Receiver operator characteristic (ROC) curve establishing a $1.7 \%$ slan+ ncMO cutoff value with an area under the ROC curve of 0.995 ; MedCalc Statistical software version 12.7 .5 (Ostend, Belgium). (E) Repeated evaluation of relative slan ${ }^{+}$ncMO percentage in ten untreated CMML patients followed from 1 to 8 months. (F) Monocyte subset repartition (right panel) and the slan ${ }^{+}$ncMO population (left panel) in a representative CMML patient at diagnosis and after 2, 4 and 8 months of follow-up, without any treatment. (G) Monocyte subset repartition (right panel) and the slan ${ }^{+} \mathrm{ncMO}$ population (left panel) in representative CMML patients before and after treatment with decitabine (6 cycles). (H) Proposed two-step algorithm for the diagnosis of CMML by flow cytometry. 
LETTERS TO THE EDITOR

Clinique, Hôpitaux universitaires Henri-Mondor (AP-HP), Créteil; 'Service d'Hématologie Clinique, Institut Bergonié, Bordeaux; ${ }^{6}$ Département d'Hématologie Clinique, Gustave Lousy Cancer Center, Villejuif; 'Service d'Hématologie Clinique, Hôpital Saint-Louis (AP-HP), Parisand ${ }^{8}$ Faculté de Médecine, Université Paris-Sud, Le Kremlin-Bicêtre, France

Funding: our research was supported by the Ligure Nationale Centre le Cancer (équipe labellisée LIGUE 2017) and grants from Institut National du Cancer (INCa) to ES (TRANSLA13-157; PRT-K16057; PRT-K16-067). The authors are indebted to patients and physicians who provided samples.

Correspondence: ORIANNE WAGNER-BALLON orianne.wagnerballon@aphp.fr or

DOROTHEA SELIMOGLU-BUET dorothee.selimoglubuet@gustaveroussy.fr doi:10.3324/haematol.2019.219782

Information on authorship, contributions, and financial \& other disclosures was provided by the authors and is available with the online version of this article at www. haematologica.org.

References

1. Orazi A, Bennett JM, Germing U, et al. Chronic myelomonocytic leukemia. WHO Classification of Tumours of Haematopoietic and Lymphoid Tissues. 2017;81-86.

2. Selimoglu-Buet D, Wagner-Ballon $O$, Saadi V, et al. Characteristic repartition of monocyte subsets as a diagnostic signature of chronic myelomonocytic leukemia. Blood. 2015;125(23):3618-3626.

3. Talati C, Zhang L, Shaken G, et al. Monocyte subset analysis accurately distinguishes CMML from $\mathrm{MDS}$ and is associated with a favorable MDS prognosis. Blood. 2017;129(13):1881-1883.

4. Patnaik MM, Sim MM, Vallapureddy $\mathrm{R}$, et al. Flow cytometry based monocyte subset analysis accurately distinguishes chronic myelomonocytic leukemia from myeloproliferative neoplasms with associated monocytosis. Blood Cancer J. 2017;7(7):e584.

5. Hudson CA, Barack WR, Leary PC, Bennett JM. Clinical utility of classical and nonclassical monocyte percentage in the diagnosis of chronic myelomonocytic leukemia. Am J Chin Pathol. 2018; 150(4):293-302.

6. Selimoglu-Buet D, Badaoui B, Benayoun E, et al. Accumulation of classical monocytes defines a subgroup of $\mathrm{MDS}$ that frequently evolves into CMML. Blood. 2017;130(6):832-835.

7. Tarfi S, Harrivel V, Dumezy F, et al. Multicenter validation of the flow measurement of classical monocyte fraction for chronic myelomonocytic leukemia diagnosis. Blood Cancer J. 2018;8(11):114.

8. Solary E, Itzykson R. How I treat chronic myelomonocytic leukemia. Blood. 2017;130(2):126-136.

9. Ziegler-Heitbrock L, Hofer TPJ. Toward a refined definition of monocyte subsets. Front Immunol. 2013;4:23.

10. Hofer TP, Zawada AM, Frankenberger M, et al. slan-defined subsets of CD16-positive monocytes: impact of granulomatous inflammatron and M-CSF receptor mutation. Blood. 2015;126(24):2601-2610.

11. Bennett JM, Catovsky D, Daniel MT, et al. The chronic myeloid leukaemias: guidelines for distinguishing chronic granulocytic, typical chronic myeloid, and chronic myelomonocytic leukaemia. Proposals by the French-American-British Cooperative Leukaemia Group. Br J Haematol. 1994;87(4):746-754.

12. Selimoglu-Buet D, Rivière J, Ghamlouch H, et al. A miR-150/TET3 pathway regulates the generation of mouse and human non-classical monocyte subset. Nat Commune. 2018;9(1):5455.

13. Wong KL, Yap WH, Tai JJY, et al. The three human monocyte subsets: implications for health and disease. Immunol Res. 2012;53(13):41-57.

14. Cargo C, Cullen M, Taylor J, et al. The use of targeted sequencing and flow cytometry to identify patients with a clinically significant monocytosis. Blood. 2019; 133(12):1325-1334.

15. Merlevede J, Droin N, Ain T, et al. Mutation allele burden remains unchanged in chronic myelomonocytic leukaemia responding to hypomethylating agents. Nat Commun. 2016;7:10767.

haematological 2020; 105:e152 